\title{
Análise de citopatologias cérvico-vaginal realizadas na Bahia entre 2015-2019:
}

\section{Indicadores Técnicos}

\author{
Analysis of cervicovaginal cytopathologies performed in Bahia between 2015-2019: Technical \\ Indicators
}

Análisis de citopatologías cervicovaginales realizadas en Bahia entre 2015-2019: Indicadores

Técnicos

Recebido: 14/09/2021 | Revisado: 20/09/2021 | Aceito: 23/09/2021 | Publicado: 25/09/2021

\author{
Bruna Lago Santos \\ ORCID: https://orcid.org/0000-0002-0733-0585 \\ Universidade Estadual do Sudoeste da Bahia, Brasil \\ E-mail: bhu.lago@hotmail.com \\ Érica Sany Brito Oliveira Costa \\ ORCID: https://orcid.org/0000-0002-6413-7650 \\ Universidade Estadual do Sudoeste da Bahia, Brasil \\ E-mail: ericasanyy @ gmail.com \\ Gabriele Dias Moreira \\ ORCID: https://orcid.org/0000-0001-8635-7067 \\ Universidade Estadual do Sudoeste da Bahia, Brasil \\ E-mail: gabys_maz@hotmail.com \\ Màyra Beatriz Alves Andrade \\ ORCID: https://orcid.org/0000-0003-3811-9878 \\ Universidade Estadual do Sudoeste da Bahia, Brasil \\ E-mail: mayrabeatrizandrade@gmail.com \\ Ninalva de Andrade Santos \\ ORCID: https://orcid.org/0000-0001-7051-7230 \\ Universidade Estadual do Sudoeste da Bahia, Brasil \\ E-mail: nsantos@uesb.edu.br \\ Thaís Pires de Melo Ximenes \\ ORCID: https://orcid.org/0000-0003-3405-6531 \\ Universidade Estadual do Sudoeste da Bahia, Brasil \\ E-mail:20170025@uesb.edu.br
}

\begin{abstract}
Resumo
Objetivo: O estudo objetivou analisar os indicadores das citopatologias cérvico-vaginal realizadas na Bahia entre 2015 e 2019. Metodologia: Estudo documental, de cunho quanti-qualitativo, do tipo descritivo que investigou indicadores técnicos do Papanicolau realizados por mulheres baianas com idade de 25 a 64 anos no período citado acima, buscando-se levantar a razão de exames realizados e proporção da periodicidade trienal e os índices de positividade e proporção de amostras insatisfatórias. A amostra foi obtida através de dados secundários do Departamento de Informática do Sistema Único de Saúde, especificamente utilizando o Sistema de Informação do Câncer, das projeções populacionais do Instituto Brasileiro de Geografia e Estatística e da Ficha Técnica de Indicadores das Ações de Controle do Câncer do Colo do Útero. Os dados obtidos foram registrados no programa Microsoft Excel 2016. Resultados: A razão de exames citopatológicos foi mais expressiva em 2018 (34,4 exames/100 mulheres/ano). A periodicidade trienal alcançou valor máximo $(96,6 \%)$ em 2017 e valor mínimo $(94,2 \%)$ em 2019. O índice de positividade teve ápice em 2019 (2,6\%). A proporção de amostras insatisfatórias foi maior em 2015 (2,4\%) com valor mínimo em 2017 (1,8\%). Considerações Finais: Os resultados deste estudo permitem inferir existirem importantes lacunas a serem consideradas para o pleno êxito do programa de prevenção do câncer de colo uterino no estado da Bahia. Assim, espera-se que esses resultados contribuam para reflexão e tomada de ações em relação às citopatologias cérvico-vaginais de modo que tenhamos, em um futuro próximo, minimização no número de casos de câncer de colo uterino.
\end{abstract}

Palavras-chave: Câncer de colo uterino; Papanicolau; Rastreamento; Indicadores.

\footnotetext{
Abstract

Objective: The study aimed to analyze the indicators of cervical-vaginal cytopathologies carried out in Bahia between 2015 and 2019. Methodology: Documentary study, of a quantitative and qualitative nature, of the descriptive type that investigated technical indicators of Pap smear performed by Bahia women aged 25 to 64 years in the period
} 
mentioned above, seeking to raise the ratio of examinations performed and the proportion of three-year periodicity and the positivity rates and proportion of unsatisfactory samples. The sample was obtained through secondary data from the Informatics Department of the Unified Health System, specifically using the Cancer Information System, population projections from the Brazilian Institute of Geography and Statistics and the Technical Data Sheet of Indicators of Cancer Control Actions Cervix of the Uterus. The data obtained were registered in the Microsoft Excel 2016 program. Results: The ratio of cytopathological tests was more expressive in 2018 (34.4 tests/100 women/year). The three-year period reached a maximum value (96.6\%) in 2017 and a minimum value (94.2\%) in 2019. The positivity index peaked in 2019 (2.6\%). The proportion of unsatisfactory samples was higher in $2015(2.4 \%)$ with a minimum value in 2017 (1.8\%). Final Considerations: The results of this study allow us to infer that there are important gaps to be considered for the full success of the cervical cancer prevention program in the state of Bahia. Thus, it is expected that these results contribute to reflection and taking actions in relation to cervical-vaginal cytopathologies so that we have, in the near future, a minimization in the number of cases of cervical cancer.

Keywords: Cervical cancer; Pap smears; Tracking; Indicators.

\section{Resumen}

Objetivo: El estudio tuvo como objetivo analizar los indicadores de citopatologías cervicovaginales realizados en Bahía entre 2015 y 2019. Metodología: Estudio documental, de carácter cuantitativo y cualitativo, de tipo descriptivo que investigó indicadores técnicos de Papanicolaou realizado por mujeres bahianas, de 25 a 64 años en el período mencionado anteriormente, buscando elevar la proporción de exámenes realizados y la proporción de periodicidad trienal y las tasas de positividad y proporción de muestras insatisfactorias. La muestra se obtuvo a través de datos secundarios del Departamento de Informática del Sistema Único de Salud, específicamente utilizando el Sistema de Información del Cáncer, proyecciones poblacionales del Instituto Brasileño de Geografía y Estadística y Ficha Técnica de Indicadores de Acciones de Control del Cáncer Cérvix de Útero. Los datos obtenidos fueron registrados en el programa Microsoft Excel 2016. Resultados: El ratio de pruebas citopatológicas fue más expresivo en 2018 (34,4 pruebas / 100 mujeres / año). El período de tres años alcanzó un valor máximo (96,6\%) en 2017 y un valor mínimo (94,2\%) en 2019. El índice de positividad alcanzó su punto máximo en 2019 (2,6\%). La proporción de muestras insatisfactorias fue mayor en $2015(2,4 \%)$ con un valor mínimo en 2017 (1,8\%). Consideraciones finales: Los resultados de este estudio permiten inferir que existen importantes brechas a considerar para el pleno éxito del programa de prevención del cáncer cervicouterino en el estado de Bahía. Así, se espera que estos resultados contribuyan a la reflexión y la toma de acciones en relación a las citopatologías cérvico-vaginales para que tengamos, en un futuro próximo, una minimización en el número de casos de cáncer de cuello uterino.

Palabras clave: Cáncer de cuello uterino; Papanicolau; Seguimiento; Indicadores.

\section{Introdução}

O câncer de colo uterino é, mundialmente, o quarto tipo de neoplasia que acomete a população feminina, de acordo com o Instituto Nacional do Câncer (INCA). No Brasil, excluindo-se o câncer de pele não melanoma, constitui o terceiro tumor maligno mais frequente, depois do câncer de mama e colorretal, sendo a quarta causa de óbito por câncer entre as mulheres (INCA, 2020; INCA, 2017). Aproximadamente 570.000 casos e 311.000 mortes devido ao câncer de colo uterino ocorreram no mundo em 2018 (Mesquita, et al., 2020). Nota-se haver uma tendência de manutenção do número de casos, visto que em 2020, mais de meio milhão de mulheres foram diagnosticadas com a patologia, sendo que 342.000 evoluíram para óbitos. Reitera-se ser a maioria dessas mulheres residentes em países em desenvolvimento ou pouco desenvolvidos, fato que chama atenção para os aspectos socioeconômicos como fatores agravantes da problemática, conforme a Organização Mundial de Saúde (OMS). No Brasil, a incidência se aproximou de 15,4 casos para cada 100 mil mulheres entre 2018 e 2019 , o que teve como desdobramento o surgimento de, aproximadamente, 16.370 novos casos (INCA, 2017; OMS, 2021). A estimativa para cada ano do triênio 2020/2022 é que sejam diagnosticados 16.590 novos casos de câncer de colo do útero no Brasil, com um risco considerado de 15,4 casos para cada 100 mil mulheres, fato que mantém o país na terceira posição do ranking mundial, contribuindo para a ascendência no quantitativo de casos em relação ao triênio anterior (INCA, 2020).

O diagnóstico do câncer cervical é mais frequente na faixa etária entre 50 e 60 anos. Todavia, considerando ser a história natural da doença, precedida por lesões pré-cancerígenas de evolução lenta, é aceitável a inferência que seu desenvolvimento tem início aos 30 anos de idade. O principal fator de vulnerabilidade para este tipo de câncer é a infecção pelo papiloma vírus humano (HPV) que está presente em 99,7\% dos casos. As alterações iniciais, denominadas Lesão 
intraepitelial de baixo grau (LIE I) ou Neoplasia Intracelular grau I (NIC-I) são normalmente transitórias, podendo regredir, espontaneamente, em $90 \%$ das mulheres infectadas. No entanto, é fundamental o adequado monitoramento dessas alterações identificadas, dadas as possibilidades de haver presença e persistência do vírus HPV (principalmente os tipos 16 e 18 que possuem maior potencial de oncogenicidade) em $10 \%$ dos casos de resultados alterados. Neste sentido, considerando que o desenvolvimento do tumor maligno possui janela temporal de 10 a 20 anos, entre o aparecimento das primeiras atipias celulares até a evolução para o câncer instalado, é profícuo afirmar que este tipo de câncer pode ser evitado através da realização periodicamente adequada do exame citopatológico cérvico-vaginal conhecido como Papanicolau (Mesquita, et al., 2020).

O Papanicolau constitui prática de rastreamento segura, de simples realização e baixo custo preconizado no Brasil para a prevenção ou deteç̧ão precoce da doença. A citologia oncótica consiste no esfregaço de células provenientes da ectocérvice e da endocérvice extraídas por raspagem do colo uterino (Moreira \& Carvalho, 2020). A amostra classificada como satisfatória deve conter células provenientes do epitélio escamosas, glandulares e metaplásicas, ou seja, da zona de transformação, em quantidades representativas e com boa distribuição, afixadas e coradas, permitindo uma segura conclusão diagnóstica no momento da leitura da lâmina (Santos \& Ribeiro, 2020).

A Organização Mundial da Saúde (OMS), bem como o Ministério da Saúde (MS), preconizam a cobertura por esse método de rastreamento a todas as mulheres na faixa etária entre 25 a 64 anos que já tenham iniciado a atividade sexual (OMS, 2013; Brasil, 2010). A recomendação a esse intervalo etário específico é justificada pelo fato de que, antes dos 25 anos, as infecções por HPV tendem a regredir espontaneamente. Por outro lado, após os 65 anos, o risco de desenvolvimento cancerígeno cervical é reduzido devido à lenta evolução do agravo (Moreira \& Carvalho, 2020). Atualmente os protocolos de realização do Papanicolau orientam que o exame seja feito, inicialmente, por dois anos consecutivos. A partir daí, caso tenham-se resultados negativos para risco carcinogênico, a repetição é indicada a cada três anos (OMS, 2013; Brasil, 2010).

Há de se considerar evidências epidemiológicas que demonstram que países que possuem uma cobertura superior a 50\% do exame Papanicolau apresentam taxas inferiores a três mortes por cem mil mulheres anualmente enquanto que nações com cobertura superior a 70\% possuem índices de mortalidade duplicadas (Brasil, 2013). Essa constatação remete a necessidade de se refletir acerca das organizações dos serviços para realização do Papanicolau. Quando a prática do exame é realizada de forma oportunista (demanda espontânea), "as mulheres são atendidas de acordo com os seus desejos, entendimentos e de forma espontânea, não havendo uma organização ou planejamento para a oferta desse serviço". Essa conduta é inadequada porque, apesar do serviço apresentar uma boa cobertura, a realização não contempla todas as mulheres que têm precisão de fazer o exame. Assim, enquanto umas não tem o devido acesso, outras repetem o exame desnecessariamente (Silva \&, 2017, p.110).

O Ministério da Saúde preconiza como meta, no mínimo, cobertura de exames citopatológicos para $80 \%$ das mulheres que se encontram na faixa etária considerada prioridade, conforme descrito anteriormente. Reitera-se a importância de que estes exames atendam os padrões de qualidade pré-estabelecidos, fato que assegura que as chances de ocorrência de câncer de colo uterino diminuam entre 60 a 90\%. Nesta perspectiva, considerando a prevalência alta de câncer de colo uterino (CCU), um importante marcador de qualidade dos programas de prevenção do agravo é o Papanicolau. Assim, a periodicidade entre a realização dos exames, a preparação da mulher, a qualidade da coleta e/ou da leitura das lâminas e o monitoramento das alterações significativas identificadas são ferramentas relevantes a serem consideradas (Santos \& Ribeiro, 2020).

A Citopatologia é o instrumento mais adequado para o rastreamento do câncer de colo uterino devido a sua praticidade e baixo custo, por isso é oferecido pela rede pública e privada de saúde (Moreira \& Carvalho, 2020). Contudo, não pode ser considerado de alta acurácia devido a sua baixa sensibilidade (taxa de falsos negativos que varia entre 6\% a $56 \%$ ) a 
qual, comumente, está relacionada aos processamentos e às análises das amostras durante o procedimento de coloração, no escrutínio e/ou interpretação (Santos \& Ribeiro, 2020).

Um importante fator que pode gerar um resultado falso-negativo, dentre outros acima citados, é a não representatividade na amostra coletada (lâmina) da Zona de Transformação, que contém o epitélio metaplásico e glandular, sendo analisado apenas o epitélio escamoso. Esse fator está relacionado ao indicador de qualidade do exame, visto que $90 \%$ das lesões precursoras se localizam na zona de transformação, e a sua ausência no esfregaço pode comprometer o diagnóstico precoce (Ceolin \&, 2020). Por outro lado, a limitação do material coletado relacionado à amostra insatisfatória está relacionada com apresenta de: sangue, infiltrado leucocitário, áreas espessas, dessecamento, artefatos de estiramento, lubrificante e contaminação, consoante indicação no Sistema Bethesda (Santos \& Ribeiro, 2020).

Na região nordeste, com exceção dos tumores de pele não melanoma, o CCU é o segundo mais incidente (INCA, 2019). Na Bahia, segundo estimativas do Instituto Nacional do Câncer para o ano de 2020, a taxa bruta de incidência da doença foi estimada em 13,85 por 100 mil mulheres (INCA, 2019).

Neste sentido, preocupa-nos, sobremaneira, a problemática referida pela Sociedade Brasileira de Cirurgia Oncológica (SBCO) e Sociedade Brasileira de Patologia (SBP), destacando que mais de 50 mil brasileiros não receberam resultados de exames diagnóstico de câncer entre março e maio de 2020, devido à pandemia causada pelo Sars-Cov 2. Esse fato foi considerado essencial para a exclusão dos dados publicados em 2020 para este estudo (SBCO, 2020). Esta constatação é relevante por serem os cânceres, em geral, tempo dependentes, ou seja, quanto mais tardio for o diagnóstico, pior o prognóstico. Assim, acreditamos ser oportuna a inferência de serem esses resultados serviços essenciais e que, portanto, não deveriam ser interrompidos de modo que o tratamento pudesse ser iniciado o mais precocemente possível.

Neste sentido, o estudo teve como objetivo analisar os indicadores das citopatologias cérvico-vaginal realizadas na Bahia entre 2015 e 2019.

\section{Metodologia}

Trata-se de pesquisa descritiva que analisou indicadores técnicos de exames citopatológicos cérvico-vaginal realizados em mulheres residentes no estado da Bahia no período de 2015 a 2019 com idade entre 25 e 64 anos. Utilizou-se dados secundários do Departamento de Informática do Sistema Único de Saúde (DATASUS), especificamente utilizando o Sistema de Informação do Câncer (SISCAN), das projeções populacionais do Instituto Brasileiro de Geografia e Estatística (IBGE) e da Ficha Técnica de Indicadores das Ações de Controle do Câncer do Colo do Útero.

Os indicadores técnicos utilizados para a análise de dados do exame citopatológico cérvico-vaginal possibilitaram avaliação quantitativa e qualitativa segundo: razão de exames realizados, proporção da periodicidade trienal, índice de positividade para alterações oncológicas e proporção de amostras consideradas insatisfatórias. O cálculo matemático de todas as variáveis encontra-se disponível no Quadro 1.

A oferta de exames, como preconizado pelo Sistema de Informação do Pacto pela Saúde (SISPACTO), foi quantificada pelo indicador razão de exames citopatológicos realizados e população-alvo feminina considerando-se o mesmo período e a mesma faixa etária. Esse cálculo fornece o alcance dos exames em relação à população total, permitindo comparação com a meta proposta segundo a Secretaria de Saúde da Bahia (SESAB) nos Relatórios Anuais de Gestão (RAG). No período de 2015 a 2017, os RAG propuseram como meta o mesmo valor para a razão de exames: 0,4 ou 40 exames/100 mulheres/ano. Nos anos de 2018 e 2019, entretanto, por não ter sido explicitada a meta pactuada, considerou-se, neste estudo, o mesmo valor da série histórica anterior.

Para o cálculo desses indicadores, na coleta de dados no SISCAN, iniciou-se selecionando "Cito do colo por local de residência" e, em seguida, restringiu-se os dados ao estado da Bahia. As seleções "coluna", "medidas" e "seleções disponíveis" 
variaram de acordo com o indicador de interesse, exceto pela "linha", a qual se manteve a opção "Faixa etária" complementada em "seleções disponíveis" com o intervalo de " 25 até 64 anos", e pelo "período disponível" em que marcou-se o intervalo entre "2015 a 2019" em todas as coletas.

Para o cálculo do Índice de Positividade, em "medidas" foi selecionada a variável "Exames alterados", sendo, para os demais indicadores, utilizada somente a variável "Exames". Na "coluna", para o numerador do indicador razão de exames citopatológicos, foi selecionado "Motivo do exame" e para o cálculo da periodicidade trienal foram feitas duas coletas com as opções "Motivo do exame" e "Período do preventivo", sendo os demais cálculos realizados com a coluna não ativa. Para o "período do preventivo", especificou-se em "seleções disponíveis", inicialmente, "mesmo ano, 1 ano, 2 anos e 3 anos" e, sem seguida, "mesmo ano, 1 ano, 2 anos, 3 anos, 4 anos ou mais" para o cálculo do indicador de periodicidade trienal. Já para a proporção de amostras insatisfatórias, também foram realizadas duas coletas diferentes em "seleções disponíveis", onde foi selecionado "adequabilidade satisfatória" e, em outro momento, "adequabilidade insatisfatória".

Por fim, visando a quantificação da população feminina baiana nos períodos supracitados para o cálculo da razão de exames citopatológicos, utilizou-se, como preconizado pelo SISPACTO, a projeção demográfica disponibilizada pelo IBGE, a qual especificava o número total de mulheres e as porcentagens referentes às faixas etárias de 25 a 64 anos desse sexo (IBGE, 2020).

Os dados obtidos foram registrados no programa Microsoft Excel 2016 de forma a facilitar a aplicação dos cálculos desejados para melhor sistematização dos resultados.

Quadro 1 - Métodos para cálculos dos indicadores, descrição da avaliação do indicador e respectivas fontes secundárias.

\begin{tabular}{|c|c|c|c|}
\hline Indicador Técnico & Cálculo (Numerador/Denominador) & $\begin{array}{c}\text { Método de } \\
\text { avaliação do } \\
\text { exame } \\
\text { citopatológico }\end{array}$ & Fonte \\
\hline $\begin{array}{c}\text { Razão de exames } \\
\text { citopatológicos do colo do } \\
\text { útero em mulheres de } 25 \text { a } \\
64 \text { anos e a população } \\
\text { feminina da mesma faixa } \\
\text { etária }\end{array}$ & $\begin{array}{l}\text { Número de exames citopatológicos do colo } \\
\text { do útero em mulheres da faixa etária de } 25 \text { a } \\
64 \text { anos residentes em determinado local e } \\
\text { ano / (Número de mulheres de } 25 \text { a } 64 \text { anos } \\
\text { residentes no respectivo local e ano / 3) }\end{array}$ & Quantitativo & SISCAN $^{\mathrm{a}}$ e IBGE ${ }^{\mathrm{b}}$ \\
\hline $\begin{array}{l}\text { Proporção de exames } \\
\text { citopatológicos do colo do } \\
\text { útero com periodicidade } \\
\text { trienal }\end{array}$ & $\begin{array}{l}\text { Número de exames citopatológicos do colo } \\
\text { do útero com relato de exame anterior no } \\
\text { período de } 3 \text { anos (mesmo ano, } 1 \text { ano, } 2 \\
\text { anos, } 3 \text { anos) em mulheres na faixa etária de } \\
25 \text { a } 64 \text { anos residentes em determinado } \\
\text { local e ano x } 100 \text { / Número de exames } \\
\text { citopatológicos do colo do útero com relato } \\
\text { de exame anterior em mulheres de } 25 \text { a } 64 \\
\text { anos residentes no respectivo local e ano }\end{array}$ & Quantitativo & SISCAN $^{\mathrm{a}, \mathrm{b}}$ \\
\hline $\begin{array}{l}\text { Índice de Positividade de } \\
\text { exames citopatológicos do } \\
\text { colo do útero }\end{array}$ & $\begin{array}{c}\text { Número de exames citopatológicos com } \\
\text { resultados alterados em determinado local e } \\
\text { período x } 100 \text { / número de exames } \\
\text { citopatológicos satisfatórios no respectivo } \\
\text { local e período }\end{array}$ & Qualitativo & SISCAN $^{\mathrm{a}, \mathrm{b}}$ \\
\hline $\begin{array}{c}\text { Proporção de amostras } \\
\text { insatisfatórias em exames } \\
\text { citopatológicos }\end{array}$ & $\begin{array}{l}\text { Número total de exames citopatológicos } \\
\text { insatisfatórios / número total de exames }\end{array}$ & Qualitativo & SISCAN $^{\mathrm{a}, \mathrm{b}}$ \\
\hline
\end{tabular}

${ }^{a}$ numerador; ${ }^{b}$ denominador. Legenda: Sistema de Informação do Câncer (SISCAN); Instituto Brasileiro de Geografia e Estatística (IBGE). Fonte: Produzido pelo autor (2020) a partir da Ficha Técnica de Indicadores das Ações de Controle do Câncer do Colo do Útero (2014) 


\section{Resultados}

A razão de exames citopatológicos realizados e a população feminina alvo apresentou crescimento significativo entre os anos de 2015 a 2018, período que foi alcançado o valor máximo de 34,36 exames/100 mulheres/ano. Entre 2018 e 2019, houve discreta queda nestes índices (Tabela 1).

Tabela 1 - Razão de prevalência do exame citopatológico cérvico-vaginal entre mulheres de 25 a 64 anos de 2015 a $2019 .{ }^{1}$

\begin{tabular}{c|c|c|c}
\hline Ano & $\begin{array}{c}\text { Citologias } \\
\text { realizadas }\end{array}$ & População alvo & Razão exames citopatológicos / população alvo \\
\hline $\mathbf{2 0 1 5}$ & 258.207 & $3.862 .060,75$ & $20,0 \%$ \\
\hline $\mathbf{2 0 1 6}$ & 334.837 & $3.919 .207,69$ & $25,6 \%$ \\
\hline $\mathbf{2 0 1 7}$ & 384.944 & $3.972 .139,45$ & $29,1 \%$ \\
\hline $\mathbf{2 0 1 8}$ & 461.079 & $4.024 .588,04$ & $34,4 \%$ \\
\hline $\mathbf{2 0 1 9}$ & 463.573 & $4.075 .163,09$ & $34,1 \%$ \\
\hline
\end{tabular}

Fonte: SISCAN e IBGE (2020).

Nas tabelas 2 e 3 apresentaremos o indicador complementar periodicidade trienal e a sua relação com a razão de exames realizados. Na sequência histórica do período analisado nota-se que a periodicidade alcançou valores máximo (96,6\%) em 2017 e mínimo (94,2\%) em 2019 mantendo o padrão de variações destacado na Tabela 1, ou seja, crescente entre 2015 a 2017 e decrescente entre 2018 e 2019. A prevalência periódica trienal do exame (recomendada pelo MS) explicita a quantidade de exames trienais/100 mulheres/ano, demonstrando que grande parte (mais de 96\%) dos exames citopatológicos entre o período de 2015 a 2019 foram realizados periodicamente, ou seja, de 1 a 3 anos.

Tabela 2 - Demonstração de exames realizados com relato anterior nos últimos três anos e daqueles com registro anterior independente do intervalo de tempo, ambos os grupos na faixa etária de 25 a 64 anos e no período de 2015 a $2019 .{ }^{2}$

\begin{tabular}{c|c|c|c}
\hline Ano & $\begin{array}{c}\text { Exames com relato de } \\
\text { registro anterior (3 anos ou } \\
\text { menos) }\end{array}$ & $\begin{array}{c}\text { Número de exames com } \\
\text { relato de registro anterior }\end{array}$ & $\begin{array}{c}\text { Periodicidade } \\
\text { Trienal }\end{array}$ \\
\hline $\mathbf{2 0 1 5}$ & 198.242 & 206.134 & $96,17 \%$ \\
\hline $\mathbf{2 0 1 6}$ & 258.247 & 268.309 & $96,24 \%$ \\
\hline $\mathbf{2 0 1 7}$ & 302.257 & 312.985 & $96,57 \%$ \\
\hline $\mathbf{2 0 1 8}$ & 355.280 & 374.391 & $94,89 \%$ \\
\hline $\mathbf{2 0 1 9}$ & 362.744 & 384.951 & $94,23 \%$ \\
\hline
\end{tabular}

Fonte: SISCAN (2020).

\footnotetext{
${ }^{1}$ Os dados utilizados para o seu cálculo: número total de exames realizados em pacientes dessa mesma faixa etária e o terço da população de mulheres estimada pelo IBGE.

${ }^{2} \mathrm{Na}$ última coluna, o indicador técnico de periodicidade trienal resultante do quociente entre as duas primeiras variáveis
} 
Tabela 3 - Porcentagem equivalente à prevalência periódica trienal do exame citopatológico cérvico-vaginal em mulheres de 25 a 64 anos.

\begin{tabular}{c|c|c|c}
\hline Ano & $\begin{array}{c}\text { Razão do exame citopatológico } \\
\text { cérvico-vaginal como } \\
\text { rastreamento } \\
\text { (exames/mulheres) }\end{array}$ & $\begin{array}{c}\text { Periodicidade } \\
\text { Trienal }\end{array}$ & $\begin{array}{c}\text { Prevalência periódica } \\
\text { trienal da CCV }\end{array}$ \\
\hline 2015 & $20,0 \%$ & $96,2 \%$ & $19,2 \%$ \\
\hline 2016 & $25,6 \%$ & $96,2 \%$ & $24,6 \%$ \\
\hline 2017 & $29,1 \%$ & $96,6 \%$ & $28,1 \%$ \\
\hline 2018 & $34,4 \%$ & $94,9 \%$ & $32,6 \%$ \\
\hline 2019 & $34,1 \%$ & $94,2 \%$ & $32,1 \%$ \\
\hline
\end{tabular}

Fonte: SISCAN (2020).

O índice de positividade, ou seja, detecção de alterações celulares de relevância para a oncologia, indicador avaliado na Tabela 4, apresentou crescimento ao longo dos anos, tendo, em 2019, o maior valor obtido no período (2,6\%). A mais expressiva alteração ocorreu entre 2016 e 2017, intervalo onde ocorreu crescimento de $0,2 \%$. A menor variação aconteceu entre os anos de 2018 e 2019 (0,04\%).

Tabela 4 - Índice de positividade do exame citopatológico cérvico-vaginal entre mulheres de 25 e 64 anos.

\begin{tabular}{c|c|c|c}
\hline Ano & $\begin{array}{c}\text { Exames considerados } \\
\text { satisfatórios }\end{array}$ & $\begin{array}{c}\text { Exames com resultado } \\
\text { alterado }\end{array}$ & Índice de positividade \\
\hline 2015 & 251.565 & 4.890 & $1,9 \%$ \\
\hline 2016 & 327.836 & 6.885 & $2,1 \%$ \\
\hline 2017 & 377.094 & 9.206 & $2,4 \%$ \\
\hline 2018 & 451.248 & 11.572 & $2,6 \%$ \\
\hline 2019 & 452.041 & 11.788 & $2,6 \%$ \\
\hline
\end{tabular}

Fonte: SISCAN (2020).

A Tabela 5 evidencia que, em 2015, a proporção de amostras insatisfatórias atingiu o valor máximo dentre o período estudado (2,3\%). Entre os anos de 2015 a 2017, nota-se decréscimo no quantitativo de exames realizados. Porém, há de se considerar a manutenção, com tendência a aumento de amostras insatisfatórias no ano de 2019 (2,2\%). 
Tabela 5 - Proporção de exames citopatológico cérvico-vaginal com amostras insatisfatórias para a análise.

\begin{tabular}{|c|c|c|c|}
\hline Ano & $\begin{array}{c}\text { Total de } \\
\text { exames }\end{array}$ & $\begin{array}{c}\text { Exames } \\
\text { Insatisfatórios }\end{array}$ & $\begin{array}{c}\text { Proporção Amostras } \\
\text { Insatisfatórias }\end{array}$ \\
\hline 2015 & 258.207 & 6.104 & 2,36 \\
\hline 2016 & 334.837 & 6.212 & 1,85 \\
\hline 2017 & 384.944 & 6.912 & 1,79 \\
\hline 2018 & 461.079 & 8.571 & 1,85 \\
\hline 2019 & 463.573 & 10.241 & 2,20 \\
\hline
\end{tabular}

Fonte: SISCAN (2020).

\section{Discussão}

Nota-se que a razão de exames citopatológicos realizados pelo Sistema Único de Saúde (SUS) em mulheres de 25 a 64 anos residentes na Bahia, no período de 2015 a 2017, apresentou-se abaixo do recomendado pela meta estadual proposta para o período (40\%). Nos anos de 2018 e 2019, não foram explicitados nos RAG as metas objetivadas desse indicador. Neste estudo, por serem utilizadas as projeções do IBGE, a razão calculada não se iguala aos resultados apresentados pela SESAB nos Relatórios de Gestão. No entanto, há de se considerar que, apesar dessa diferença de valores, a razão permaneceu abaixo da meta pactuada.

Neste sentido, ratifica-se a relevância de ações que possibilitem elevar a cobertura do exame a exemplo da difusão de informações para que as mulheres reconheçam a importância da adesão ao procedimento, assegurar condições relacionadas com estrutura física, recursos humanos e materiais necessários para a realização qualificada da citopatologia (Santos \&, 2019).

O conhecimento da razão de exames citopatológicos do colo do útero pode constituir importante ferramenta para correlacionar oferta e demanda, na identificação de situações de desigualdade e tendências que demandem ações e estudos específicos, bem como subsidiar processos de planejamento, gestão e avaliação de políticas públicas na área de saúde da mulher. Todavia, há de se considerar ser um viés desse indicador a identificação da oferta de exame citopatológico com base no número de exames realizados e não no quantitativo de mulheres examinadas o que, por sua vez, pode não retratar a real cobertura da população alvo do rastreamento levando a pseudo impressão de boa cobertura. Ademais, o SISCAN disponibiliza dados relacionados, somente, com os exames realizados pela rede conveniada ao SUS fato a ser considerado dado que possivelmente interfere na transparência da análise (Brasil, 2013).

Resultados falsos-negativos na rotina de é uma condição a ser considerada. Assim, o protocolo do Ministério da Saúde recomenda que o exame citopatológico seja realizado trienalmente após dois exames com resultados sem alterações significativas realizados com intervalo de um ano (INCA, 2016). Resultados cujas amostras foram classificadas como insatisfatória ou que apresentaram alterações de interesse no campo da oncologia deverão ter condutas de seguimento diferenciado de acordo com as necessidades identificadas. Esse protocolo se alinha com diretrizes de países com programa de rastreamento organizado que tiveram resultados efetivos na prevenção do câncer de colo uterino (OMS, 2007).

Estudos têm evidenciado que predomina no Brasil o modelo de rastreamento oportunístico no qual a maioria das mulheres realiza exames através da demanda espontânea (frequência maior do que o necessário) ceifando a oportunidade que outras mulheres da população alvo sejam examinadas, fato imprescindível para o êxito das ações de prevenção dessa neoplasia (Damacena \&, 2017). Destaca-se que na Bahia, no período analisado a realização do exame citopatológico trienal esteve dentro do recomendado com valores acima de 94\%, ou seja, superior aos 85\% estabelecidos pelo INCA como meta (INCA, 2014). No entanto, inquieta-nos a percepção de não haver uma diminuição correspondente nos índices de câncer de colo uterino no 
estado. Essa constatação permite uma inferência: será que o problema está no seguimento adequado dos casos alterados identificados ou na qualidade da leitura das lâminas que podem levar a resultados falso negativos? Acreditamos serem estas questões importantes a serem consideradas.

Outro indicador importante é o índice de positividade, o qual expressa a prevalência de alterações celulares de interesse caracterizando a sensibilidade do rastreamento em detectar lesões precursoras de neoplasia de colo uterino na população examinada (Araujo, et al., 2015). A análise desse indicador, neste estudo, foi considerada insatisfatória, visto que os dados não estiveram entre 3\% a 10\%, conforme o estabelecido pelo Ministério da Saúde (Machado, et al., 2018). A baixa positividade pode indicar que amostras de interesse significativo não estão sendo identificadas pelos laboratórios responsáveis pelas leituras das lâminas (exames falso-negativos). Essa realidade é preocupante porque, como já dito anteriormente, algumas mulheres poderão ter câncer de colo de útero apesar de realizarem o exame preventivo várias vezes (INCA, 2016). A falsanegatividade pode estar associada a erro de coleta, erro de escrutínio ou erro de interpretação. Assim,

o erro de coleta ocorre pela não representatividade da junção escamo-colunar (JEC), sobreposição celular e hipocelularidade no esfregaço. O erro de escrutínio dá-se pela não identificação e reconhecimento de células neoplásicas representadas no esfregaço, seja por falta de atenção e concentração, tempo insuficiente para analisar o esfregaço, fadiga mental ou pouca experiência do profissional. $\mathrm{O}$ erro de interpretação sucede quando as células neoplásicas são reconhecidas, mas são interpretadas como benignas ou subavaliadas e classificadas erroneamente, seja pela inexperiência do citopatologista ou por informações clínicas inadequadas (Machado et al, 2018, pp. 244-245).

Ademais, pode estar associado ao erro de coleta a existência de esfregaços dessecados, presença de sangue, secreção purulenta e/ou de fundo necrótico, além de apresentação com áreas espessas (Rezende, 2016).

A análise do percentual de amostras insatisfatórias é fundamental por permitir a quantificação de exames que não apresentam condições mínimas de leitura da lâmina em relação ao total de exames feitos (Damacena \&, 2017). Neste estudo observa-se que os dados alcançaram o aceitável preconizado pela Organização Mundial da Saúde (abaixo de 5\%). Todavia, há de se considerar ser a meta nacional índice de amostra insatisfatória abaixo de 1\% (Santo \&, 2012). Apesar de ter ocorrido um decréscimo da proporção entre os anos de 2015 a 2017, houve um aumento significativo até o ano de 2019, chegando ao valor de 2,2, o que evidencia a persistência de erros desde a coleta até a conservação do produto.

Segundo a Nomenclatura Brasileira para laudos citopatológicos cervicais, a qual é adotada pelo Sistema de Informação do Controle de Câncer do Colo Uterino (SISCOLO), a amostra pode ser considerada insatisfatória na fase préanalítica pela ausência de identificação da paciente na amostra e/ou forma de requisição, lâmina quebrada ou outras causas alheias ao laboratório (Galvão \&, 2015). Dessa forma, para a amostra alcançar a qualidade ideal, deve apresentar células escamosas, glandulares e metaplásicas em quantidade representativa, fixadas e coradas, de maneira que seja possível realizar uma conclusão diagnóstica (Rodrigues \&, 2016; INCA, 2016).

Neste sentido reitera-se ser imprescindível o controle de qualidade relacionado com as leituras das lâminas de modo a detectar, corrigir e reduzir as deficiências do processo de produção minimizando a ocorrência de erros diagnósticos (INCA, 2016). Capacitação de recursos humanos e programas de educação continuada são fundamentais para aperfeiçoar o desempenho geral, assim como a aplicação do teste de proficiência para a devida qualidade do procedimento (INCA, 2016). Além disso, o controle de qualidade em citopatologia do colo do útero também está associado com a atribuição de limites de carga de trabalho por citologista, levando-se em conta que o citologista possui outras tarefas diárias no laboratório além da leitura de rotina (Tobias, 2016). 


\section{Considerações Finais}

O estudo permitiu avaliar a importância da análise dos indicadores de citopatologias cérvico-vaginais para o programa de prevenção do câncer de colo uterino de acordo com os objetivos propostos. Este tipo de câncer, que possui considerável número de casos pode ser efetivamente evitado com a realização adequada da citopatolologia, fato que reitera a importância de estudos que contribuam para avaliação dessa estratégia.

Há de se considerar os vários fatores que interferem no quantitativo e/ou na qualidade do método de rastreamento, a exemplo do conhecimento das mulheres acerca da importância do exame, bem como da sua apropriada preparação para a citologia, coleta adequada com representação de amostras celulares de interesse, boa preparação e fixação da lâmina, correta leitura e interpretação dos resultados (considerar resultados falso-positivos e falso negativos) além do devido seguimento dos casos com resultados de interesse no campo da oncologia. Ademais, o rastreamento organizado deve ser considerado dado que vários estudos têm sinalizado haver no Brasil predominância de realizações de metas atingidas de forma através da demanda espontânea.

Os resultados deste estudo permitem inferir que existem importantes lacunas a serem consideradas e sanadas para o pleno êxito do programa de prevenção do câncer de colo uterino no estado da Bahia. Nota-se haver necessidade de se fortalecer a tríade que envolve ações educativas, capacitação profissional e adequação institucional (recursos matérias e organização das demandas) para o êxito do programa.

Diante dessas considerações, espera-se que os resultados deste estudo possam contribuir para reflexão e tomada de ações em relação às citopatologias cérvico-vaginais de modo que se tenha, em um futuro próximo, minimização no número de casos de câncer de colo uterino.

\section{Referências}

Araujo Jr, M. L. C., Santana, D. A., Almeida, L. B., Quintana, S., Silva, G. R. F., \& Fonseca, R. C. (2015). Qualidade em citopatologia: análise de indicadores de monitoramento interno de qualidade do Instituto Nacional de Câncer. Jornal Brasileiro de Patologia e Medicina Laboratorial,51(2), 102-107. http://www.dx.doi.org/10.5935/1676-2444.20150018.

Ceolin, R., Nasi, C., Coelho, D. F., Paz, A. A., \& Lacchini, A. J. B. (2020). Análise do rastreamento do câncer do colo do útero de um município do sul do Brasil. Rev. Pesqui. (Univ. Fed. Estado Rio J., Online), 12(1), 440-446. http://seer.unirio.br/index.php/cuidadofundamental/article/viewFile/8342/pdf_1.

Damacena, A. M., Luz, L. L., \& Mattos, I. E. (2017). Rastreamento do câncer do colo do útero em Teresina, Piauí: estudo avaliativo dos dados do Sistema de Informação do Câncer do Colo do Útero, 2006-2013. Epidemiologia e Serviços de Saúde, 26(1), 71-80. https://doi.org/10.5123/S1679-49742017000100008.

Galvão, E. F. B., da Silva, M. J. M., Esteves, F. A. M., \& Peres, A. L. (2015). Frequência de amostras insatisfatórias dos exames preventivos do câncer de colo uterino na rede pública de saúde, em município do agreste pernambucano. Rev Para Med,29(2), 51-6. http://files.bvs.br/upload/S/01015907/2015/v29n2/a5012.pdf

Instituto Brasileiro de Geografia e Estatística, (2020). Projeção da população do Brasil e das Unidades da Federação. https://www.ibge.gov.br/apps/populacao/projecao/index.html?utm_source=portal\&utm_medium=popclock\&utm_campaign=novo_popclock

Instituto Nacional de Câncer José Alencar Gomes da Silva, (2013). Sistema de Informação do Câncer: manual preliminar de apoio à implantação. Rio de Janeiro. https://www.saude.sc.gov.br/index.php/documentos/atencao-basica/saude-da-mulher/siscan/7171-manual-preliminar-siscan/file

Instituto Nacional de Câncer José de Alencar Gomes da Silva, (2013). Monitoramento das ações de controle dos cânceres do colo do útero e de mama. https://bvsms.saude.gov.br/bvs/publicacoes/inca/Informativo_Deteccao_Precoce_agosto_dezembro_2012.pdf.

Instituto Nacional de Câncer José Alencar Gomes da Silva, (2014). Ficha técnica de indicadores das ações de controle do câncer do colo do útero. https://www.inca.gov.br/sites/ufu.sti.inca.local/files//media/document//fichatecnicaindicadorescolo14.pdf.

Instituto Nacional de Câncer José Alencar Gomes da Silva, (2016). Diretrizes Brasileiras para o Rastreamento do Câncer do Colo do Útero, (2a ed.), http://www.citologiaclinica.org.br/site/pdf/documentos/diretrizes-para-o-rastreamento-do-cancer-do-colo-do-utero_2016.pdf.

Instituto Nacional de Câncer José Alencar Gomes da Silva, (2016). Manual de Gestão da Qualidade para Laboratório de Citopatologia, (2a ed.), http://www.citologiaclinica.org.br/site/pdf/documentos/diretrizes-para-o-rastreamento-do-cancer-do-colo-do-utero_2016.pdf.

Instituto Nacional de Câncer José Alencar Gomes da Silva, (2019). Estimativa 2020: incidência de câncer no Brasil. https://www.inca.gov.br/publicacoes/livros/estimativa-2020-incidencia-decancer-no-brasil. 
Instituto Nacional de Câncer José Alencar Gomes da Silva, (2017). Estimativa 2018: incidência de câncer no Brasil. https://rbc.inca.gov.br/revista/index.php/revista/article/view/115.

Instituto Nacional de Câncer José Alencar Gomes da Silva, (2020). Estimativa 2020-2022: incidência de câncer no Brasil. https://www.inca.gov.br/numerosde-cancer/estimativa-triênio-2020-2022

Machado, E. P., Alves, M. B. M., Irie, M. M. T., Zrzebiela, F. F., Reche, P. M., \& Borato, D. C. K. (2018). Controle interno de qualidade em citopatologia: o dilema da subjetividade. Rev. bras. anal. clin, 53(1), 244-249. http://www.rbac.org.br/artigos/controle-interno-de-qualidade-em-citopatologia-o-dilema-dasubjetividade/.

Mesquita, A. D. D., Teles, K. K. N., Silva, S. C. B. D., Silva, F. R. D., Lima, L. K. C., Costa, R. S. L. D., \& Arruda, E. F. D. (2020). Conhecimentos, atitudes e práticas de mulheres frente ao exame preventivo do câncer do colo uterino. J. Health NPEPS, 5(1), 261-275. https://periodicos.unemat.br/index.php/jhnpeps/article/view/4184.

Ministério da Saúde, (2010). Caderno de Atenção Primária, n. 29. https://bvsms.saude.gov.br/bvs/publicacoes/cadern o_atencao_primaria_29_rastreamento.pdf.

Ministério da Saúde, (2013). Controle dos cânceres do colo do útero e da mama, (2a ed.), https://ufsj.edu.br/portal-repositorio/File/lapsam/APA__ANPAD.pdf.

Moreira, A. P. L., \& Carvalho, A. T. D. (2020). Tendência de Realização da Citologia Oncótica e Fatores Associados em Mulheres de 25 a 64 anos. Rev. bras. ciênc. saúde, 24(1), 17-28. https://periodicos.ufpb.br/index.php/rbcs/article/view/46938.

Organização Mundial da Saúde. (2013). WHO guidance note: comprehensive cervical cancer prevention and control: a healthier future for girls and women. https://apps.who.int/iris/handle/10665/78128.

Organização Mundial da Saúde. (2007). Cancer control: Knowledge into action: WHO guide for effective programmes. Module 3, Module 3. World Health Organization. http://catalog.hathitrust.org/api/volumes/oclc/219104041.html

Organização Mundial da Saúde. (2020). Nota de orientação da OPAS/OMS: HPV e Câncer do Colo do Útero. https://www3.paho.org/pt/topicos/hpv-e-cancerdo-colo-do-utero?page=2

Rezende, M. T. (2016). Comparação dos exames citopatológicos do colo do útero do município de Ouro Preto-MG, submetidos ao monitoramento externo da qualidade. http://www.repositorio.ufop.br/jspui/handle/123456789/7924.

Rodrigues, J. F., Moreira, B. A., Alves, T. G. S., \& de Azevedo Guimarães, E. A. (2016). Rastreamento do câncer do colo do útero na região ampliada oeste de Minas Gerais. Revista de Enfermagem do Centro-Oeste Mineiro, 6(2), 2156-2168.

Santos, M. J. S., \& Ribeiro, A. A. (2020). Estratégias Utilizadas para Melhorar a Qualidade dos Exames Citopatológicos. Revista Brasileira de Cancerologia, 66(1), e-05104. https://doi.org/10.32635/2176-9745.RBC.2020v66n1.104.

Santos, R. D. S., Melo, E. C. P., \& Santos, K. M. (2012). Análise espacial dos indicadores pactuados para o rastreamento do câncer do colo do útero no Brasil. Texto \& Contexto-Enfermagem, 21(4), 800-810. https://www.scielo.br/j/tce/a/RhYf7dHpHR79sddtdTdLgZp/abstract/?lang=pt.

Santos, T., Silveira, M., \& Rezende, H. (2019). A Importância do Exame Citopatológico na Prevenção do Câncer do Colo Uterino. ENCICLOPÉDIA BIOSFERA, 16(29), 1947-1961. https://ares.unasus.gov.br/acervo/handle/ARES/9185.

Secretaria Estadual de Saúde da Bahia, (2020). Relatório anual de Gestão 2017. http://www.saude.ba.gov.br/wp-content/uploads/2020/06/Relat\%C3\%B3rioAnual-de-Gest\%C3\%A3o-2017.pdf.

Secretaria Estadual de Saúde da Bahia, (2020). Relatórios anuais de Gestão. http://www.saude.ba.gov.br/sobre-a-sesab/relatorios-anuais-de-gestao-rag/.

Silva, A. B., Rodrigues, M. P., de Oliveira, A. P., \& de Melo, R. H. V. (2017). Prevenção do câncer cervicouterino: uma ação realizada pelos enfermeiros da estratégia saúde da família? Revista Ciência Plural, 3(2), 99-114. https://periodicos.ufrn.br/rcp/article/view/12926/8993.

Sociedade Brasileira de Cirurgia Oncológica, (2020). Sociedades médicas apontam redução de $70 \%$ das cirurgias e que 50 mil brasileiros não receberam diagnóstico de câncer. http://sbco.org.br/2020/05/14/sociedades-medicas-apontam-reducao-de-70-das-cirurgias-e-que-50-mil-brasileiros-nao-receberamdiagnostico-de-cancer/.

Tobias, A. H. G. (2016). Desempenho do pré-escrutínio rápido e da revisão rápida de 100\% como métodos de monitoramento interno da qualidade dos exames citopatológicos do colo do útero. Tese de doutorado, Universidade Federal de Ouro Preto, Ouro Preto, MG, Brasil. http://www.repositorio.ufop.br/jspui/handle/123456789/6517. 